\title{
UTILIZAÇÃO DO SOFTWARE DE DIAGNOSE DA COMPOSIÇÃO NUTRICIONAL (CND) PARA AVALIAÇÃO DO ESTADO NUTRICIONAL DO CITROS
}

Luis Eduardo Vieira Pinto, José Eduardo Creste, Fernando Bernardo Martins, Alline Mendes Alves, Alexandre Paião Leite da Silva, Felipe Silva Loosli

Universidade do Oeste Paulista - UNOESTE, Programa de Pós-Graduação em Agronomia, Presidente Prudente, SP. Email: levp@unoeste.br

\section{RESUMO}

A citricultura tem se colocado como importante atividade para o setor agrícola e a economia brasileira nas últimas décadas. A compreensão da distribuição de nutrientes na árvore é de suma importância para o estabelecimento de programas de manejo nutricional eficientes para a produção de citros. A análise foliar é uma das técnicas utilizadas para a avaliação do estado nutricional das plantas, e sua interpretação possibilita verificar a ocorrência de deficiências, toxidez ou desequilíbrio de nutrientes. O método CND é uma técnica utilizada para diagnosticar o estado nutricional das plantas, baseia-se nas interações múltiplas que ocorrem entre todos os nutrientes sob diagnose. $O$ trabalho foi desenvolvido em área experimental da Universidade do Oeste Paulista, com o objetivo de avaliar o estado nutricional do citros, laranja pera sobre limão cravo, de um pomar de 12 anos de idade instalado na área. O método (CND) foi eficaz na avaliação nutricional do citros.

Palavras-chave: Citricultura. Nutrientes. Análise foliar. Deficiência. Fertilidade.

USE OF NUTRITIONAL COMPOSITION DIAGNOSIS (CND) SOFTWARE FOR CITROS NUTRITIONAL STATUS

\begin{abstract}
Citriculture has become an important activity for the agricultural sector and the Brazilian economy in the last decades. The understanding of the distribution of nutrients in the tree is of paramount importance for the establishment of efficient nutritional management programs for citrus production. Foliar analysis is one of the techniques used to evaluate the nutritional status of plants, and its interpretation makes it possible to verify the occurrence of nutrient deficiencies, toxicity or imbalance. The CND method is a technique used to diagnose the nutritional status of plants, based on the multiple interactions that occur among all the nutrients under diagnosis. The objective of this study was to evaluate the nutritional status of citrus, pear orange on lemon clove, from a 12 - year - old orchard in the area. The method (CND) was effective in the nutritional evaluation of citrus.
\end{abstract}

Keywords: Citriculture. Nutrients. Leaf analysis. Deficiency. Fertility.

\section{INTRODUÇÃO}

No Brasil as frutas cítricas foram introduzidas pelas primeiras expedições colonizadoras provavelmente na Bahia, quando o país foi dividido pela Coroa Portuguesa em capitanias hereditárias, que trouxeram novos imigrantes (ABECITRUS, 2017).

A laranja Pêra, Citrus sinensis L. Osbeck, pertence à família Rutaceae, sub-tribo Citrinae. Três gêneros têm importância econômica: Citrus, Fortunella e Poncirus. Caracteriza-se como árvore de porte médio, galhos mais ou menos eretos e folhas acuminadas. Sua produção atinge 
em média $250 \mathrm{~kg}$ de frutos por planta. Os frutos têm forma ovalada, com três a quatro sementes e peso médio de 145 gramas; a casca é de cor alaranjada, de espessura fina a média, quase lisa e com vesículas de óleo em nível. Tem polpa de cor laranja viva e textura firme, com suco abundante (SIMÃO, 1971). O destino do fruto é para consumo ao natural, nos mercados internos e externos ou para suco concentrado, é considerada a variedade mais plantada no Estado de São Paulo (OLIVA, 2002).

Atualmente, em área de 386 mil hectares, distribuídos em pomares jovens e adultos, são produzidos anualmente 364 milhões de caixas de laranja com uma produtividade média de 2,09 caixas por árvore. Desse total, cerca de 125 mil hectares são destinados para a variedade Pera Rio, com uma densidade média de 495 árvores por hectare, com uma produtividade média de 1,9 caixas por árvore e 913 caixas por hectare, totalizando uma produção de cerca de 114 milhões de caixas de laranja da variedade (FUNDECITRUS, 2017).

O acompanhamento do estado nutricional das culturas frutíferas, através da análise de folhas, permite a adequada gestão da nutrição mineral dos pomares, empregando fertilizantes e corretivos em quantidades adequadas para satisfazer as necessidades das plantas. É importante destacar que as técnicas de diagnóstico (solo e planta) não se excluem mutuamente, sendo complementares. Há diversas técnicas de avaliação do estado nutricional, podendo-se destacar, conforme proposto por Malavolta (2006), as seguintes: a) diagnose visual; b) diagnose foliar; c) testes bioquímicos; d) técnicas de infiltração; e) medição indireta de clorofila; f) bioavaliação; g) análise de outros órgãos. Os resultados de análises químicas do tecido vegetal podem ser interpretados por diversos métodos, sobressaindo-se os métodos univariados, como o teor crítico (TC) e a faixa de suficiência (FS), bivariados, como o sistema integrado de diagnose e recomendação (DRIS), e o multivariado, como a diagnose da composição nutricional (CND) (ROZANE et al., 2016).

O conceito CND expressa a composição mineral dos tecidos vegetais na forma de teor de nutrientes ou valores relativos, que é a informação numérica básica para estabelecer o diagnóstico do estado nutricional das plantas. O método emprega a análise composicional e a análise de componentes principais dos dados, tendo, pois, potencial mais elevado para melhorar a sensibilidade do diagnóstico da cultura em estudo. O CND vem sendo utilizado para avaliação do estado nutricional de várias culturas comerciais, em diferentes regiões do País (POLITI et al., 2013; DIAS et al., 2013; PARTELLI et al., 2014).

O método da Diagnose da Composição Nutricional (CND) também tem sido utilizado para a definição de valores de referência (Khiari et al., 2001a,b) e difere do DRIS pelo fato de o teor de cada nutriente na amostra (xi) ser corrigido em função da média geométrica da composição nutricional (G), resultando na variável multinutriente (Vi), conforme Parent e Dafir (1992) e Parent et al. (1993). Em seguida, ela é estudentizada, ou seja, calcula-se o índice da variável multinutriente (Ivi), a partir da diferença entre o valor de $\mathrm{Vi}$ da amostra e a respectiva média para a população de referência (vi), dividida pelo desvio padrão desta variável [lvi = (Vi - vi)/svi]. Em suma, além da diferença no enfoque no equilíbrio nutricional, bivariado e multivariado, para o DRIS e o CND, respectivamente, estes métodos distinguem-se pelo fato de que, no primeiro, é calculada a média aritmética das relações duais transformadas em variáveis normais reduzidas. No CND, calcula-se a média geométrica do produto dos teores de nutrientes e de um valor de complemento (denominado $\mathrm{R}$ ), para depois se proceder à transformação em variável normal reduzida. Contudo, apesar do método CND basear-se nas interações múltiplas que ocorrem entre todos os nutrientes sob diagnose, também se considera que o nutriente está em equilíbrio nutricional quando o índice da variável multinutriente (Ivi) é igual a zero.

Em tese, o método CND mostra-se vantajoso por considerar a interação simultânea de todos os nutrientes, e não apenas as interações duais como a de outros métodos existentes. E isto, 
aliado a um melhor embasamento estatístico, quando se usa princípios da análise do componente principal, propicia maior potencial para melhorar a diagnose foliar (Parent e Dafir, 1992; Parent et al. 1994).

O objetivo do trabalho é fazer o uso do software CND na cultura do citros, afim de avaliar de forma qualitativa a condição nutricional da planta, através da interpretação dos resultados analíticos obtidos. A partir disso poder compreender o estado nutricional de uma planta específica ou de várias plantas em condições semelhantes, auxiliando na recomendação de adubação para a cultura, devendo esta ser mais equilibrada, reduzindo custos e impacto ambiental, mas sem perder o objetivo de incrementar a produtividade.

\section{METODOLOGIA}

O trabalho foi desenvolvido em área experimental da Universidade do Oeste Paulista, situada no município de Presidente Prudente - SP. O clima da região se classifica em Aw, com estação chuvosa no verão e estação seca no inverno. A área monitorada no experimento é composta por um pomar de laranja Pera (Citrus sinensis L. Osbeck), enxertados em limoeiro 'Cravo' (C. limonia Osbeck) com 12 anos de idade e espaçamento de 6,6 x 2,8 metros, totalizando 525 plantas por hectare.

As características do solo, na camada de $0-20 \mathrm{~cm}$, no pomar apresentou os seguintes valores: matéria orgânica de 24,3 $\mathrm{g} \mathrm{dm}^{-3} ; \mathrm{pH}\left(\mathrm{CaCl}_{2}\right)$ 7,3; $\mathrm{P}$ (resina) $161 \mathrm{mg} \mathrm{dm}^{-3} ; \mathrm{S} 35,6 \mathrm{mg} \mathrm{dm}^{-3}$; $\mathrm{H}+\mathrm{Al}$ e $\mathrm{K}$, Ca e Mg trocáveis de 8,0; 9,2; 58,2 e 39,6 mmolc $\mathrm{dm}^{-3}$, respectivamente, $\mathrm{V}$ de 93,0\%, Fe, $\mathrm{Cu}, \mathrm{Mn}, \mathrm{Zn}$ e B, respectivamente, de 30,$1 ; 1,7 ; 1,4 ; 3,5$ e $0,21 \mathrm{mg} \mathrm{dm}^{-3}$.

Por se tratar de um trabalho no qual o objetivo não era efetuar nenhum tipo de comparação entre tratamentos, optou-se por realizar a seleção de uma planta de forma arbitrária da região central do pomar, levando em consideração que a mesma fosse saudável para que não houvesse interferência na realização da diagnose foliar. Foram coletadas todas as folhas existentes na planta e divididas entre folhas jovens e folhas adultas, considerando como folha adulta aquela fisiologicamente ativa, com brotação na primavera anterior, ou seja, com idade acima de 7 meses na época da coleta. Metodologia que se distingue do que normalmente é realizado na coleta convencional de amostras foliares para determinação da análise de tecido vegetal, porém respeitando a época recomendada de coleta das folhas. Convencionalmente a coleta baseia-se na retirada apenas da 3a ou 4a folha a partir do fruto, gerada na primavera, com cerca de 6 meses de idade, quando o fruto tem 2-4 cm de diâmetro. O material vegetal coletado foi acondicionado em sacos de papel Kraft e foram levados para o laboratório, onde foram lavados, secos em estufa de circulação forçada de ar com temperatura de $65^{\circ} \mathrm{C}$, moídos e submetidos à análise quanto aos teores totais de N, P, K, Ca, Mg, S, B, Cu, Fe, Mn e Zn (CARMO et al., 2000).

De posse dos resultados, utilizou-se o software CND-Citros (Programa de computador) da Universidade Estadual Paulista "Júlio de Mesquita Filho" - Campus de Registro, Universidade Federal do Ceará e Université Laval para a execução dos cálculos matemáticos e a interpretação dos resultados obtidos. O conceito CND expressa a composição mineral dos tecidos vegetais na forma de teor de nutrientes ou valores relativos, que é a informação numérica básica para estabelecer o diagnóstico do estado nutricional das plantas. O método emprega a análise composicional e a análise de componentes principais dos dados, tendo, pois, potencial mais elevado para melhorar a sensibilidade do diagnóstico da cultura em estudo.

\section{RESULTADOS}

O conhecimento do equilíbrio nutricional das plantas é importante para que se possa avaliar o potencial de rendimento e buscar o equilíbrio nutricional. A avaliação do estado nutricional de plantas por meio dos métodos de diagnose foliar, os quais relacionam os teores de 
nutrientes entre si, como no caso da Diagnose da Composição Nutricional (CND), vem se destacando dos tradicionais, que consideram os teores dos nutrientes individualmente, como o nível crítico e a faixa de suficiência.

Tabela 1. Resultados de análise de tecido vegetal de macro e micronutrientes das folhas de laranja Pera.

\begin{tabular}{|c|c|c|c|c|c|c|c|c|c|c|c|}
\hline & $\mathrm{N}$ & $P$ & $\mathrm{~K}$ & $\mathrm{Ca}$ & $\mathrm{Mg}$ & $S$ & $B$ & $\mathrm{Cu}$ & $\mathrm{Fe}$ & $\mathrm{Mn}$ & $\mathrm{Zn}$ \\
\hline & \multicolumn{6}{|c|}{$\mathrm{g} \mathrm{kg}^{-1}$} & \multicolumn{5}{|c|}{$\mathrm{mg} \mathrm{kg}^{-1}$} \\
\hline FOLHAS JOVENS & 22 & 1,76 & 5,3 & 27,7 & 3 & 1,6 & 31 & 13,3 & 219,5 & 15,6 & 43,3 \\
\hline FOLHAS ADULTAS & 17,7 & 1,49 & 5,4 & 29,4 & 2,8 & 1,9 & 32,5 & 12,2 & 230,7 & 14,7 & 18,7 \\
\hline
\end{tabular}

Fonte: Laboratório de Nutrição de Plantas - Unoeste.

Tabela 2. Índice da variável multinutriente (CND).

\begin{tabular}{lccccccccccc}
\hline & $\mathrm{IN}$ & $\mathrm{IP}$ & $\mathrm{IK}$ & $\mathrm{ICa}$ & $\mathrm{IMg}$ & $\mathrm{IS}$ & $\mathrm{IB}$ & $\mathrm{ICu}$ & $\mathrm{IFe}$ & $\mathrm{IMn}$ & $\mathrm{IZn}$ \\
\hline & \multicolumn{1}{c}{} & \multicolumn{1}{c}{ Índices (CND) } \\
\cline { 2 - 11 } FOLHAS JOVENS & 1,68 & 4,51 & $-2,36$ & 0,67 & 1,03 & $-0,79$ & $-2,34$ & $-1,64$ & 4,28 & $-2,18$ & 1,26 \\
FOLHAS ADULTAS & 0,74 & 4,06 & $-1,89$ & 1,61 & 1,12 & 1,27 & $-1,93$ & $-1,63$ & 4,88 & $-2,09$ & $-0,77$ \\
\hline
\end{tabular}

Fonte: http://www.registro.unesp.br/sites/cnd citros/

Figura 1. Gráficos do índice da variável multinutrientes (CND), folhas jovens (esquerda) e folhas adultas (direita).
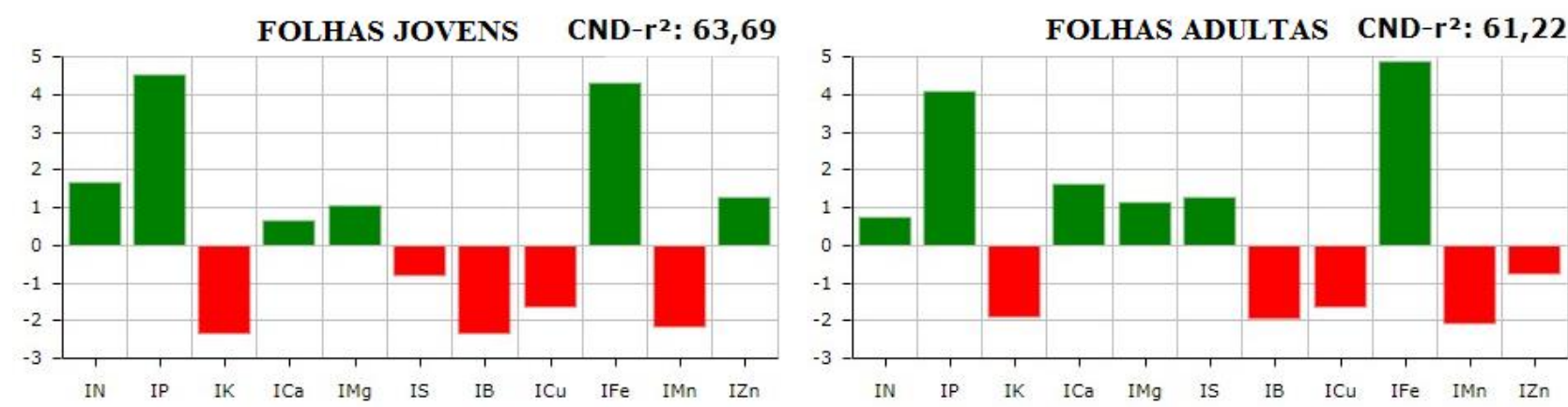

Fonte: http://www.registro.unesp.br/sites/cnd citros/

\section{DISCUSSÃO}

Pode-se interpretar através dos resultados expressos na tabela 2 e figura 1 que, em relação à população de alta produtividade, todo índice na cor verde está em "excesso", como o Nitrogênio $(\mathrm{N})$, Fósforo (P), Cálcio (Ca), Magnésio (Mg), Ferro (Fe) e Zinco (Zn), e que todo índice na cor vermelha está em "deficiência", como o Potássio (K), Enxofre (S), Boro (B), Cobre (Cu) e Manganês (Mn). Por outro lado, podem ser considerados como "adequados" os índices que tendem a zero, a exemplo do Cálcio (Ca) nas folhas jovens.

O nitrogênio é um componente-chave de enzimas, vitaminas, clorofila e outros constituintes celulares, os quais são essenciais para o crescimento e desenvolvimento das plantas. Portanto, o nitrogênio é um dos nutrientes essenciais mais importantes para a obtenção de altas produtividades de citros. O nitrogênio desempenha papel essencial no desenvolvimento dos citros, influindo no crescimento, na floração, na produção e na qualidade dos frutos, conforme ampla revisão feita por Lovatt et al. (1992). O nitrogênio é, depois do cálcio, o elemento mais extraído pela parte vegetativa da laranjeira (MARCHAL e LACOEUILHE, 1969). Entretanto, a exportação de nutrientes pelos frutos sofre modificações, com o nitrogênio sendo o elemento mais exportado, depois do potássio. Legaz et al. (1982) estudaram a absorção e distribuição do nitrogênio, a partir de adubo marcado, em Citrus. Verificaram que $26 \%$ do nutriente se acumulava 
nos órgãos mais velhos (folhas e ramos), 35\% nos órgãos mais novos e o restante nas raízes. Esses dados condizem com os obtidos no experimento, quando pode-se notar que o índice de nitrogênio é maior nas folhas jovens do que quando comparado com as folhas adultas.

Segundo Ortega e Malavolta (2012) o excesso de fósforo na planta, pode gerar uma ação de antagonismo em relação a absorção de boro e cobre, bem como o excesso de ferro pode gerar o antagonismo na absorção de cobre, manganês e zinco. O antagonismo ocorre quando a presença de um íon diminui a absorção de outro, independentemente da concentração do íon na solução do solo. Isso pode ser observado tanto nos índices das folhas jovens quanto das folhas adultas, com exceção apenas do zinco nas folhas jovens.

A produtividade e qualidade da produção de citros é amplamente influenciada pela adubação potássica (CANTARELLA et al., 2003; ALVA et al., 2006). O potássio apresenta funções importantes no metabolismo e na existência dos vegetais como: ativador enzimático, regulador osmótico, influi sobre relações hídricas, presença no transporte de carboidratos, manutenção de equilíbrio eletroquímico nas células e efeito marcante sobre extensibilidade celular que, consequentemente, modifica tamanho e qualidade interna dos frutos (LAVON et al., 1995). Os índices baixos de potássio nas folhas podem ter ocorrido devido a redistribuição do mesmo para os frutos, segundo Malavolta (2005) esse nutriente move-se facilmente para outros órgãos como folhas novas, frutos e grãos, os quais atuam como forte dreno fisiológico, justificando o fato deste elemento ser facilmente redistribuído de um órgão de residência para outro órgão de formação principalmente em plantas com idade avançada. De acordo com Mattos Jr. et al., (2003) junto com o nitrogênio, o potássio é o nutriente mais exportado pelos frutos de laranja.

\section{CONCLUSÃO}

Os elementos fósforo e ferro apresentaram maior limitação por excesso, enquanto o potássio, boro, cobre e manganês apresentaram maior limitação pela falta dos elementos na planta.

Nas folhas adultas há um maior equilíbrio do elemento nitrogênio, os índices de cálcio e magnésio estão muito próximos do adequado, principalmente nas folhas mais novas.

O método da Diagnose da Composição Nutricional (CND) foi eficaz na avaliação nutricional do citros, diagnosticando um desbalanceamento nutricional na planta.

\section{REFERÊNCIAS BIBLIOGRÁFICAS}

ALVA, A.K.; PARAMASIVAM, S.; OBREZA, T.A.; SCHUMANN, A.W. Nitrogen best management practice for citrus trees. I. Fruit yield, quality, and leaf nutritional status. Scientia Horticulturae, v.107, p.233-244, 2006 https://doi.org/10.1016/i.scienta.2005.05.017

ASSOCIAÇÃO BRASILEIRA DOS EXPORTADORES DE CÍTRICOS. História da laranja. 2008. Disponível em http://www.associtrus.com.br/: Acesso em: 31 julho. 2017.

CANTARELLA, H.; MATTOS Jr., D.; QUAGGIO, J.A.; RIGOLIN, A.T. Fruit yield of Valencia sweet orange fertilized with different $\mathrm{N}$ sources and the loss of applied $\mathrm{N}$. Nutrient Cycling in Agroecosystems., v. 67, p. 205-213, 2003 https://doi.org/10.1023/B:FRES.0000003600.20499.76

CARMO, C.A.F. de S. do; ARAÚJO, W.S. de; BERNARDI, A.C. de C.; SALDANHA, M.F.C. Métodos de análise de tecidos vegetais utilizados pela Embrapa Solos. Rio de Janeiro: Embrapa Solos, 2000. 41p. 
DIAS, J. R. M.; TUCI, C. A. F.; WADT, P. G. S.; PARTELLI, F. L.; PEREZ, D. V.; ESPINDULA, M. C.; TOMIO, D. B. Antecipação do período de diagnose foliar em laranjeira 'pera' no Amazonas. Pesquisa Agropecuária Brasileira, v. 48, p. 757- 764, 2013. Disponível em: < http://dx.doi.org/10.1590/S0100-204X2013000700008>.

FUNDECITRUS (Fundo de Defesa da Citricultura). Sumário Executivo da Estimativa da safra de Laranja. $2017 . \quad$ Disponível em: http://www.fundecitrus.com.br/pdf/pes relatorios/2017 1005 Sumario-Executivo-daEstimativa-da-Safra-de-Laranja-2017-2018.pdf Acesso em: 31 julho. 2017.

KHIARI, L.; PARENT, L.E. \& TREMBLAY, N. Critical compositional nutrient indexes for sweet corn at early growth stage. Agron. J., 93:809-814, 2001a. https://doi.org/10.2134/agronj2001.934809x

KHIARI, L.; PARENT, L.E. \& TREMBLAY, N. The phosphorus compositional nutrient diagnosis range for potato. Agron. J., 93:815-819, 2001b. https://doi.org/10.2134/agronj2001.934815x

LAVON, R.; GOLDSHMITH, E.E.; SALOMON, R.; FRANK, A. Effect of potassium, magnesium, and calcium deficiencies on carbohydrate pools and metabolism in citrus leaves. Journal of American Society of Horticultural Sciences, v.120, p.54-58, 1995.

LEGAZ, F.; PRIMO-MILLO, E.; PRIMO-YUFERA, E.; GIL, C.; RUBIO, J.L. Nitrogen fertilization in citrus. I Absortion and distribuition of nitrogen in calamondin trees (Citrus mitis $\mathrm{Bi}$ ) during flowering, fruit set and initial fruit developments periods. Plant and Soil, Dordrecht, v.66, p.339-351, 1982. https://doi.org/10.1007/BF02183800

LOVATT, C.L.; SAGEE, O.; ALI, A.G.; ZHENG, Y. Influência do nitrogênio, carboidratos e reguladores de crescimento de plantas no florescimento, frutificação e produção de citros. In: SEMINÁRIO INTERNACIONAL

DE CITROS, 2., 1992. Anais... p.27-42.

MALAVOLTA, E. Manual de nutrição mineral de plantas. São Paulo: Editôra Agronômica Ceres. 2006. $638 \mathrm{p}$.

MALAVOLTA, E. Potássio - Absorção, transporte e redistribuição na planta. In: YAMADA,T.; ROBERTS, T. L. (Ed). Potássio na agricultura brasileira. Piracicaba: POTAFÓS, 2005. P. 179-238.

MARCHAL, J.; LACOEUILHE, J.J. Bilan minéral du mandarinier 'Wilking'. Influence de la production et de l'état végétatif de l'arbre sur sa composition minérale. Fruits, Paris, v.24, p.299- 318,1969.

MATTOS JR, D.; QUAGIO, J. A.; CANTARELLA, H.; ALVA, A. K. Nutrient contente of biomass components of Hamlin sweet orange tess. Sci. Agric., 60: 155-160. 2003. https://doi.org/10.1590/S0103-90162003000100023

OLIVA, B. P. Influência das variedades cítricas (Citrus sinensis L. Osbeck) Natal, PêraRio, e Valência na qualidade do suco de laranja pasteurizado. 2002. 162 p. Tese (Doutorado em Tecnologia de Alimentos)-Faculdade de Engenharia de Alimentos, Universidade de Campinas, Campinas, 2002. 
ORTEGA, A. E.; MALAVOLTA, E. Los más recientes micronutrientes vegetales. Informaciones Agronómicas de Hispanoamérica, Georgia, v. 7, n. 1, p. 16-25, 2012.

PARENT, L.E. \& DAFIR, M. A theoretical concept of compositional nutrient diagnosis. J. Am. Soc. Hortic. Sci., 117(2):239-242, 1992.

PARENT, L. E.; ISFAN, D.; TREMBLAY, N.; KARAM, A. Multivariate nutrient diagnosis of the carrot crop. Journal of the American Society of Horticultural Science, Mount Vernon, v.119, n. 3, p. 420426, 1994.

PARENT, L.E.; KARAM, A. \& VISSER, S.A. Compositional nutrient diagnosis of the greenhouse tomato. Hortic. Sci., 28(10):1041-1042, 1993.

PARTELLI, F. L.; DIAS, J. F. M.; VIEIRA, H. D.; WADT, P. G. S.; JUNIOR, E. P. Avaliação nutricional de feijoeiro irrigado pelos métodos CND, DRIS e faixas de suficiência. Revista Brasileira Ciência do Solo, v. 38, p. 858-866, 2014. https://doi.org/10.1590/S0100-06832014000300017

POLITI, L. S.; FLORES, R. A.; SILVA, J. A. S. da; WADT, P. G. S.; PINTO, P. A. da C.; PRADO, R. de M. Estado nutricional de mangueiras determinado pelos métodos DRIS e CND. Revista Brasileira de Engenharia Agrícola e Ambiental, v. 17, p. 11 -18, 2013. https://doi.org/10.1590/S1415$\underline{43662013000100002}$

ROZANE, D. E.; PARENT, L. E.; NATALE, W. Evolution of the predictive criteria for the tropical fruit tree nutritional status. Cientifica, v. 44, n. 1, p. 102-112, 2016. https://doi.org/10.15361/1984$\underline{5529.2016 v 44 n 1 \mathrm{p} 102-112}$

SIMÃO, S. Manual de fruticultura. Piracicaba: Agronômica Ceres, 1971. 529 p. 